\title{
Optical response of grain boundaries in upgraded metallurgical-grade silicon for photovoltaics
}

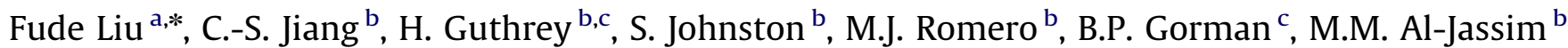 \\ a Department of Mechanical Engineering, The University of Hong Kong, Pokfulam Road, Hong Kong, China \\ ${ }^{\mathrm{b}}$ National Center for Photovoltaics, National Renewable Energy Laboratory, Golden, CO 80401, United States \\ ${ }^{\mathrm{c}}$ Department of Metallurgical and Materials Engineering, Colorado School of Mines, CO 80401, United States
}

\section{A R T I C L E I N F O}

Article history:

Received 21 December 2010

Received in revised form

6 April 2011

Accepted 21 April 2011

Available online 18 May 2011

Keywords:

Upgraded metallurgical-grade silicon

(UMG-Si)

Photovoltaics

Grain boundaries

Light emission

Impurities

Characterization

\begin{abstract}
A B S T R A C T
Using upgraded metallurgical-grade silicon (UMG-Si) is a cost-effective and energy-efficient approach for the production of solar cells. Grain boundaries (GBs) play a major role in determining the device performance of multicrystalline $\mathrm{Si}(\mathrm{mc}-\mathrm{Si})$ solar cells. In this study two UMG-Si wafers, one from the middle part of a brick and the other from the top part of the same brick, were investigated. An excellent correlation was found between the grain misorientation and the corresponding optical response of GBs as indicated by photoluminescence (PL) imaging, electron backscattered diffraction (EBSD), and crosssectional transmission electron microscopy (TEM). In addition, the PL features at random GBs depend also on the impurity levels in the wafer. In particular the PL emission was greatly enhanced in the narrow regions close to the random GB in the top wafer, which is an interesting phenomenon that may have potential application in high efficiency light-emission diodes (LEDs) based on Si.
\end{abstract}

(c) 2011 Elsevier B.V. All rights reserved.

\section{Introduction}

So far, solar modules made of Si wafers (multicrystalline ones in particular) have dominated the solar market. Si wafers (monocrystalline and multicrystalline) are traditionally made of electronic-grade silicon (EG-Si), which is obtained from metallurgicalgrade silicon (MG-Si) via complex purification processes [1]. Using upgraded metallurgical-grade silicon (UMG-Si) is regarded as a cost-effective and energy-efficient approach for the production of Si wafers. Since Si wafers are one of the major costs of solar modules, UMG-Si will be one of the technologies that help drive down the cost of solar energy. Although UMG-Si is three orders of magnitude less pure compared with EG-Si, it has been demonstrated that the performance of high-quality UMG-Si solar cells can be comparable to that of typical EG-Si counterparts [2]. However, the UMG-Si wafer quality needs to be further improved in order to have a high market penetration. Grain boundaries (GBs) play an important role in determining the device performance of solar cells made of multicrystalline silicon (mc-Si), because GBs enhance minority carrier recombination and reduce majority carrier transport. In addition GBs are possible shunting paths and also the preferred sites for extrinsic impurities, which is

\footnotetext{
* Corresponding author. Tel.: +852 2859 2631; fax: +852 28585415 . E-mail address: fordliu@hku.hk (F. Liu).
}

particularly so for UMG-Si because of its impurity levels. Note also that wafers sliced from the top part of ingots fabricated by the directional solidification process contain higher impurity levels, because impurity elements (except B) have distribution coefficients much less than one [1]. Therefore, it is necessary to have a systematic study of GBs and their behaviors in UMG-Si.

Photoluminescence (PL) is an important technique for measuring the defect density and lifetime in association with the material purity and crystalline quality of semiconductors [3]. Compared with other PL techniques such as PL spectroscopy and PL mapping, PL imaging has recently been demonstrated to be a rapid contactless characterizing technique with relatively high special resolution, which makes it a useful tool for studying mc-Si wafers [4,5]. On the other hand, electron backscattered diffraction (EBSD) is a technique that is often used to examine the crystallographic orientation of crystalline materials from electron-diffraction patterns [6]. Combining these two techniques will then allow us to determine the correlation between the PL behaviors and the misorientation of GBs in UMG-Si wafers. In addition, transmission electron microscopy (TEM) specimens can be prepared from specifically selected GBs using a dual-beam focused ion beam (FIB) workstation; the specimen can then be further investigated by TEM at high resolution.

We have recently investigated the structural and electronic/ electrical properties of individual GBs in polycrystalline thin films with grain sizes in micrometer order, where the depletion of 
carriers on the GBs was measured by scanning capacitance microscopy (SCM) due to its fine resolution of tens of nanometers $[7,8]$. The electrical and structural properties were well correlated. Due to the large grain sizes of mc-Si we in this paper employed PL imaging for the electrical property study, and found consistent relationship between the structural and electrical properties in these Si-based materials.

\section{Experimental}

Two UMG-Si wafers (B doped with a size of $156 \times 156 \mathrm{~mm}^{2}$ ), one sliced from the middle part of a brick and the other from the top part of the same brick, were examined. The wafers were first etched in $\mathrm{HF}(\sim 1 \%)$ to remove the native oxide, and were then submerged in a methanol-iodine solution [9]. The surface passivation can reduce surface recombination and allow for dramatically improved PL imaging of the bulk wafer. The wafers were imaged by PL imaging while they were still in the solution. All measurements were carried out at room temperature.

Based on the PL results, we selected a few typical GBs according to their different optical response. The same GBs (marked by a paper mask) were then studied by plan-view EBSD mapping to get grain misorientation between two adjacent grains. The EBSD mapping was done with an SEM microscope (Hitachi

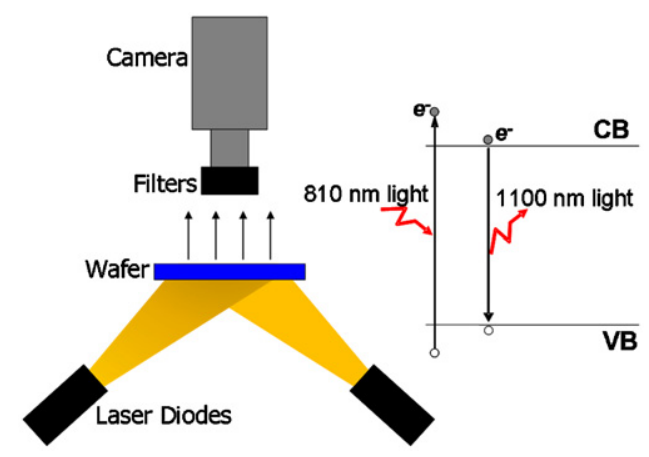

Fig. 1. Schematic of the PL imaging setup and its working principles
S-4300N) at $20 \mathrm{kV}$, a sample tilt angle of $70^{\circ}$, and a working distance of $15 \mathrm{~mm}$. In addition, specifically selected cross-sectional TEM specimens were prepared using a focused-ion-beam (FIB) workstation (FEI-Nova 200 Dual Beam) at normal operating parameters. These specimens were then further investigated using a TEM microscope (FEI-T30) at $200 \mathrm{kV}$ by bright- and dark-field imaging, selected-area diffraction (SAD), and energydispersive X-ray spectroscopy (EDX). The same procedure was applied to both the middle wafer and the top wafer.

The PL imaging setup and its working principles are illustrated in Fig. 1 . The wafer is illuminated by $810 \mathrm{~nm}$ light from the back side to avoid interference between the incident light and emitted light. Electrons are excited from the valence band to the conduction band. The electrons then relax to the conduction band edge, and some emit light at $\sim 1100 \mathrm{~nm}$ as they recombine back to the valence band. This emission passes through the filter and is detected by the Si charge-coupled device (CCD) camera. The illumination intensity is equivalent to 1 Sun, and the data acquisition time is $600 \mathrm{~s}$.

\section{Results and discussion}

The optical image, PL image, and EBSD maps of the middle wafer are shown in Fig. 2. A paper mask (hole diameter $=8 \mathrm{~mm}$ ) is used to mark the region of interest. From the optical image (Fig. 2(a)), we can see clearly the GBs. Five different GBs (1-5) and three different regions (I-III) are studied in detail as shown in Fig. 2(b) and Fig. 2(c), respectively. Although the PL image was taken from the same region as that of the optical counterpart, we can see that different GBs show different optical responses. The inset in Fig. 2(b) displays the image intensity profile from the region indicated by the rectangular frame in the PL image, where GBs with different PL intensities are marked by arrows and numbers. The EBSD maps obtained from three regions (I-III) are displayed in Fig. 2(c). The EBSD map is color-coded with the inverse pole figure (IPF) coloring in the sample normal direction $Z_{0}$. Also shown is the grain misorientation (angle and axis of rotation) between adjacent grains. $\Sigma 3 \mathrm{GBs}$ are indicated by white lines; the misorientation to all the $\Sigma 9 \mathrm{GBs}$ in this study was experimentally measured to be $\sim 39.5^{\circ}$ and $\langle 011\rangle$. Comparing the PL image and
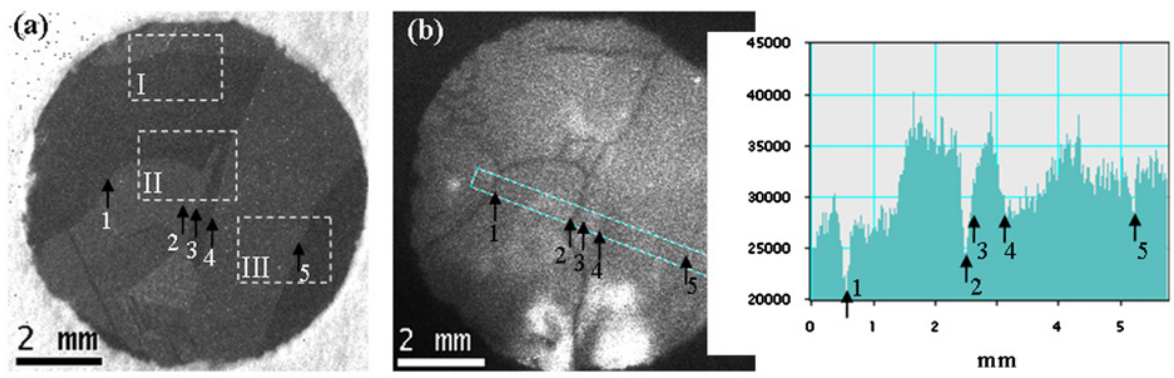

(c)
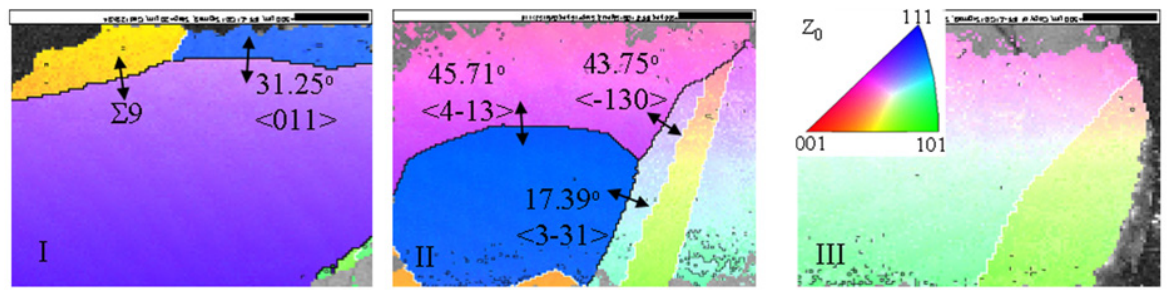

Fig. 2. Middle wafer. (a) Optical image. A paper mask (hole diameter $=8 \mathrm{~mm}$ ) is used to mark the region of interest. Five different GBs (1-5) and three different regions (I-III) are studied in detail in (b) and (c), respectively. (b) PL image of the same region as in (a). The inset displays the image intensity profile from the region indicated by the rectangular frame in the PL image. GBs with different PL intensities are indicated by arrows and numbers. (c) EBSD maps obtained from three different regions (I-III) as shown in (a). The inset in panel III is the color-coding legend. Also shown is the grain misorientation between adjacent grains ( $\Sigma 3$ GBs are indicated by white lines). (For interpretation of the references to color in this figure legend, the reader is referred to the web version of this article.) 
EBSD maps, we can see that the random boundaries show strong PL imaging contrast while the contrast at $\Sigma 3$ and $\Sigma 9$ coincidence site lattice (CSL) boundaries can barely be seen. It is well known that the intensity of radiative band-to-band recombination decreases at defective GBs, due to the dominance of non-radiative recombination centers at the defects. Because random GBs contain more defects (and more impurities also), it is not surprising that the nonradiative recombination dominates and they appear dark in the PL image. On the other hand, $\Sigma 3$ and $\Sigma 9$ GBs are much less defective. Therefore, a clear correlation exist between the grain misorientation and the corresponding optical response of GBs.

These above results are consistent with the observation that different silicon GBs have different minority carrier diffusion lengths and recombination velocities as evaluated by laser beam induced current (LBIC) [10]. In an electron beam induced current (EBIC) study, Tsurekawa et al. [11] found that the EBIC contrast is approximately 2-3 times as high at the random boundary as at $\Sigma 3$ and $\Sigma 9$ CSL boundaries in the whole range of temperatures (50-300 K). The EBIC contrast of these CSL boundaries is less than $5 \%$ at $300 \mathrm{~K}$. In addition, there is little difference among the EBIC contrasts of $\left\{\begin{array}{lll}1 & 1 & 1\end{array}\right\} \Sigma 3,\left\{\begin{array}{lll}1 & 1 & 2\end{array}\right\} \Sigma 3$, and $\left(\begin{array}{lll}1 & 1 & \overline{1}\end{array}\right) /\left(\begin{array}{lll}5 & \overline{1} & 1\end{array}\right) \Sigma 9$ boundaries. In a recent study on polycrystalline silicon (poly-Si) thin films [7] we found carrier depletions on most random GBs and some $\sum 9$ GBs, but not on the $\sum 3$ GBs. The carrier depletion could be a measure for a charged $\mathrm{GB}$ indicating that the GB has deep energy levels and/or impurity ions.

In order to investigate why random GBs and CSL GBs have different optical responses, the same middle wafer was further examined with TEM. Two GBs in the region I of Fig. 2 were selected. SEM and TEM images are shown in Fig. 3. Fig. 3(a) corresponds to the $\Sigma 9 \mathrm{~GB}$, including an SEM image (tilted for a better 3D view) and a cross-sectional TEM image. From the SEM image, we see that the GB is symmetric along the GB plane and the GB is straight. These two observations are consistent with the structure of $\Sigma 9$ boundaries. The inset is a SAD pattern from the GB at the $\langle 110\rangle$ zone axis, which further confirms that the GB is $\Sigma 9$. In contrast, the random large-angle GB shown in Fig. 3(b) is neither symmetric nor straight. In fact, many zig-zag segments were observed along the random GB when zoomed in at higher magnifications (not shown here). So it is understandable that random GBs are dark in PL images as shown in Fig. 2, because of more recombination centers caused by more structural defects and high impurity levels at the random GBs.

As mentioned before, the top wafer contains more impurities than the middle one. It is important to know the effect of impurity levels on the optical response and the device performance. The PL image is shown in Fig. 4(a). It is quite interesting to note that the GBs light up, unlike those in the middle wafer. However, the very center of the boundary is still dark although the darkness varies for different GBs as in the case of the middle wafer. This is illustrated in the inset, which is the magnified PL image from region I, with the image intensity profile from the region indicated by the arrow. The image intensity profile clearly shows the strong PL emission of the regions around the GB. The emission at some GBs can be enhanced by more than $50 \%$ compared with the grain bulk. Fig. 4(b) shows the EBSD map acquired from region II as indicated in the inset of Fig. 4(a). Again we can see a clear correlation between the grain misorientation and the corresponding optical response of GBs, by comparing the EBSD map with the PL image. Note that impurities do not have as much effect on the electrical activity of $\Sigma 3$ and $\Sigma 9$ boundaries as that on random GBs - the PL contrast of the CSL boundaries is still weak and no strong PL emission is observed around these GBs.

Silicon has traditionally been regarded as a semiconductor material with poor light emission because of its indirect bandgap. However, considerable efforts have been put forward to realize a

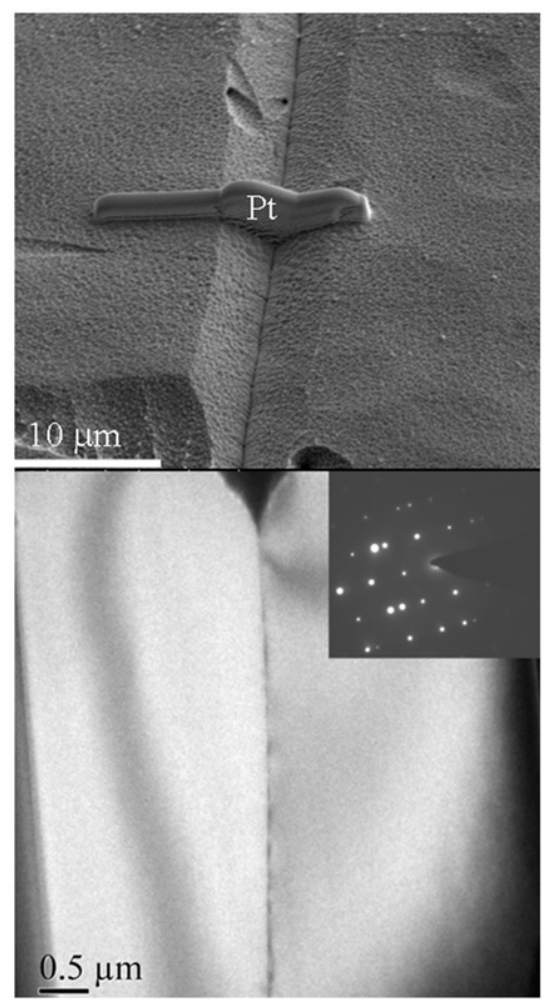

b

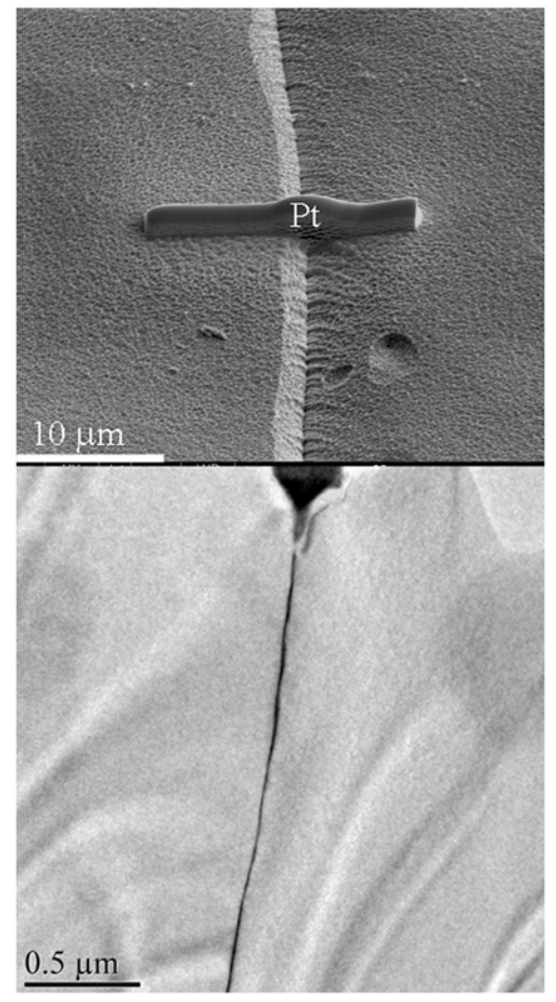

Fig. 3. Middle wafer. (a) SEM image (tilted for a better 3D view) and cross-sectional TEM image of the $\Sigma 9$ GB. The inset is a SAD pattern at the $\langle 110\rangle$ zone axis from the GB. (b) SEM image and cross-sectional TEM image of the random large-angle GB. The GBs are those two displayed in the region I of Fig. 2. 


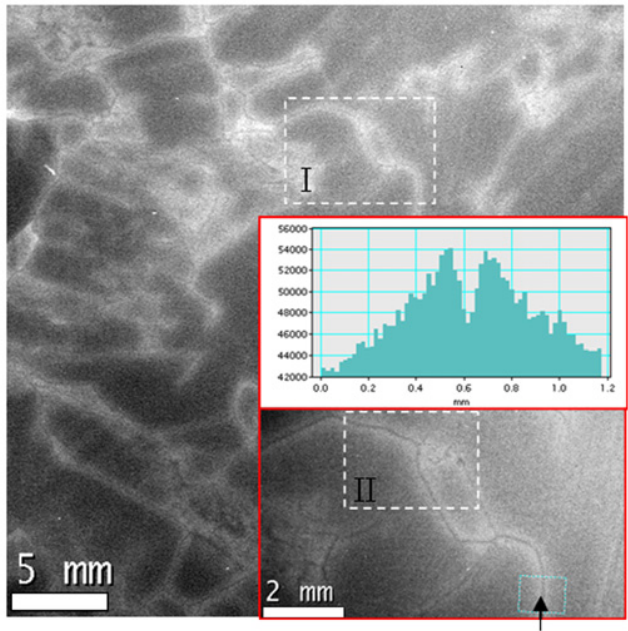

b

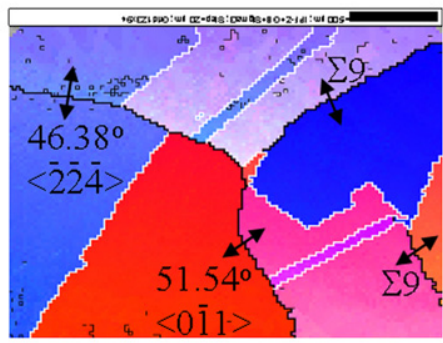

C

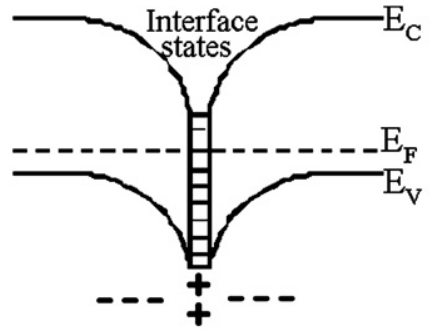

Fig. 4. Top wafer. (a) PL image. The inset is the magnified PL image from regions I and II. Also shown is the image intensity profile from the region indicated by the arrow in the inset. (b) EBSD map. The map was acquired from region II as indicated in the inset of (a). (c) GB model. The depletion regions near the GB are polarity inverted.

strong light emission of silicon at room temperature because of its important potential applications [12]. The strong PL emission around the GB in the top wafer, which contains relatively high impurities, is very interesting, and it needs further studies. Denuded zones (i.e., the bright region in the vicinity of GBs) have been observed by Chen et al. [13] with plan-view EBIC imaging in the top and bottom wafers from an mc-Si ingot. This phenomenon was attributed to the impurity gettering effect by GBs to purify the regions just near the boundary. However, we think the purifying effect is unlikely to be the main contribution considering the higher overall impurity levels and still much stronger absolute PL emission level near the GB in the top wafer than in the middle wafer (the impurity level in regions near the GB may be still higher in the top wafer than in the middle wafer). In fact, the grain bulk PL emission in both the middle wafer and the top wafer was found to be similar.

In the literature, it has been reported that the top part of an ingot could be n-type due to the close average concentrations of $\mathrm{B}$ and $\mathrm{P}$ (in the order of $\sim 10^{17} \mathrm{~cm}^{-3}$ ) and their different segregation coefficients [2,14]. Mandurah et al. [15] showed that $P$ has a strong tendency to segregate to the GBs while B does not. Itoh et al. [16] determined that the segregation ratios of the $\mathrm{P}$ concentrations in the grain bulk to those at the GBs are greater than one. Based on these facts the GBs in the top wafer can be doped with P, so that the GBs are doping- or polarity-inverted in the p-type material, forming double tiny $\mathrm{p}-\mathrm{n}$ junctions centered at the GB [Fig. 4(c)]. The radiative recombination might be enhanced in the local $p-n$ junction region due to the excess minority carriers and considerable deviation of majority carriers from the thermal equilibrium state when the wafer is illuminated by $810 \mathrm{~nm}$ light. Such a locally inverted GB has also been proposed on Si and other photovoltaic materials [17-20]. We tried to check the impurity levels at GBs with EDX using scanning TEM (STEM) mode in our microscope and found that the impurity levels are below the detection limit $(<1$ at\%). So other techniques should be applied to clarify this issue, for example, using the secondary ion mass spectrometry (SIMS) to get the composition profiling across the GB.

Another possible mechanism for the enhanced PL could be the strain field around the GBs. The strain may lead to direct bandgap $\mathrm{Si}$, which helps boost radiative recombination and eliminate phononmediated recombination [21,22]. An excellent correlation between potential change, structure, and stress at individual GBs has also been reported in the literature [23].

\section{Conclusions}

Based on the findings, we conclude that the optical response at GBs is grain-orientation dependent as indicated by the clear correlation between the PL and EBSD results: random GBs contain many recombination centers whereas $\Sigma 3$ and $\Sigma 9$ boundaries are found to be almost electrically inactive even when the impurity levels are high. In addition the PL features at random GBs depend also on the impurity levels in the wafer, which is closely related to the wafer position where it is sliced from the ingot. In particular an interesting phenomenon is observed for the first time that the PL emission is greatly enhanced in the narrow regions close to the random GBs in the top UMG-Si wafer, which may have potential application in high efficiency light-emission diodes (LED) based on Si. Overall, we believe that this study will help us with a deeper understanding on GBs and their behaviors not only in UMG-Si but also in other mc- and polycrystalline materials, for the applications in photovoltaics and other solid-state devices such as LEDs.

\section{Acknowledgments}

F. Liu thanks the financial support from the Seed Funding Programme for Basic Research at HKU (Project Code 200910159016) and the HKU Initiative on Clean Energy and Environment (HKU-ICEE) funding. At NREL, this work is supported by the US Department of Energy under Contract no. DE-AC36-08-GO28308.

\section{References}

[1] B. Ceccaroli, O. Lohne, Solar grade silicon feedstock, in: A. Luque, S. Hegedus (Eds.), Handbook of Photovoltaic Science and Engineering, Wiley, 2003, pp. 153-201.

[2] D. Kohler, B. Raabe, S. Braun, S. Seren, G. Hahn, Upgraded metallurgical grade silicon solar cells: a detailed materials analysis, in: Proceedings of the 24th EU PVSEC, Hamburg, 2009, pp. 1758-1761.

[3] D.K. Schroder, Semiconductor Material and Device Characterization, 2nd ed. Wiley, 1998, pp. 623-627. 
[4] H. Sugimoto, M. Tajima, Photoluminescence imaging of multicrystalline Si wafers during HF etching, Jpn. J. Appl. Phys. 46 (2007) L339-L341.

[5] T. Trupke, R.A. Bardos, M.C. Schubert, W. Warta, Photoluminescence imaging of silicon wafers, Appl. Phys. Lett. 89 (2006) 044107-1-044107-3.

[6] V. Randle, Theoretical framework for electron backscatter diffraction, in: A.J. Schwartz, M. Kumar, B.L. Adams (Eds.), Electron Backscatter Diffraction in Materials Science, Springer, New York, 2000, pp. 19-30.

[7] C.-S. Jiang, H.R. Moutinho, F. Liu, M.J. Romero, M.M. Al-Jassim, Carrier depletion and grain misorientations on individual grain boundaries of polycrystalline $\mathrm{Si}$ thin films, in: Proceedings of the 34th IEEE Photovoltaic Specialists Conference, Philadelphia, PA, 2009, pp. 000471-000476.

[8] C.-S. Jiang, H.R. Moutinho, B. To, P. Dippo, M.J. Romero, M.M. Al-Jassim, Probing carrier depletions on grain boundaries in polycrystalline Si thin films by scanning capacitance microscopy, Mater. Res. Soc. Symp. Proc. 1066 (2008) 87-92.

[9] B. Chhabra, C. Weiland, R.L. Opila, C.B. Honsberg, Surface characterization of quinhydrone-methanol and iodine-methanol passivated silicon substrates using X-ray photoelectron spectroscopy, Phys. Status Solidi A 208 (2011) 86-90.

[10] K. Nishioka, T. Yagi, Y. Uraoka, T. Fuyuki, Effect of hydrogen plasma treatment on grain boundaries in polycrystalline silicon solar cell evaluated by laserbeam-induced current, Sol. Energy Mater. Sol. Cells 91 (2007) 1-5.

[11] S. Tsurekawa, K. Kido, T. Watanabe, Interfacial state and potential barrier height associated with grain boundaries in polycrystalline silicon, Mater. Sci. Eng. A 462 (2007) 61-67.

[12] M.A. Green, J. Zhao, A. Wang, P.J. Reece, M. Gal, Efficient silicon light-emitting diodes, Nature 412 (2001) 805-808.

[13] J. Chen, T. Sekiguchi, S. Nara, D. Yang, The characterization of high quality multicrystalline silicon by the electron beam induced current method, J. Phys.: Condens. Matter 16 (2004) S211-S216.
[14] J. Degoulange, I. Perichaud, C. Trassy, S. Martinuzzi, Multicrystalline silicon wafers prepared from upgraded metallurgical feedstock, Sol. Energy Mater. Sol. Cells 92 (2008) 1269-1273.

[15] M.M. Mandurah, K.C. Saraswat, C.R. Helms, T.I. Kamins, Dopant segregation in polycrystalline silicon, J. Appl. Phys. 51 (1980) 5755-5763.

[16] M. Itoh, I. Aikawa, N. Hirashita, T. Ajioka, Dopant segregation at polycrystalline silicon grain boundaries in device fabrication process, in: Poroceedings of the 1989 MRS Fall Meeting, vol. 164, 1990, pp. 347-352.

[17] E. Christoffel, M. Rusu, A. Zerga, S. Bourdais, S. Noel, A. Slaoui, A twodimensional modeling of the fine-grained polycrystalline silicon thin-film solar cells, Thin Solid Films 403-404 (2002) 258-262.

[18] G. Beaucarne, S. Bourdais, A. Slaoui, J. Poortmans, Carrier collection in finegrained $\mathrm{p}-\mathrm{n}$ junction polysilicon solar cells, in: Proceedings of the 28th IEEE PVSC Conference, Anchorage, Alaska, 2000, pp. 128-133.

[19] C.-S. Jiang, R. Noufi, J.A. AbuShama, K. Ramanathan, H.R. Moutinho, J. Pankow, M.M. Al-Jassim, Local built-in potential on grain boundary of $\mathrm{Cu}(\mathrm{In}, \mathrm{Ga}) \mathrm{Se}_{2}$ thin flms, Appl. Phys. Lett. 84 (2004) 3477-3479.

[20] I. Visoly-Fisher, S.R. Cohen, K. Gartsman, A. Ruzin, D. Cahen, Understanding the beneficial role of grain boundaries in polycrystalline solar cells from single-grain-boundary scanning probe microscopy, Adv. Funct. Mater. 16 (2006) 649-660.

[21] A.T. Fiory, N.M. Ravindra, Light emission from silicon: some perspectives and applications, J. Electron. Mater. 32 (2003) 1043-1051.

[22] W.L. Ng, M.A. Lourenco, R.M. Gwilliam, S. Ledain, G. Shao, K.P. Homewood, An efficient room-temperature silicon-based light-emitting diode, Nature 410 (2001) 192-194.

[23] S. Honda, T. Mates, B. Rezek, A. Fejfar, J. Kocka, Microscopic study of the $\mathrm{H}_{2} \mathrm{O}$ vapor treatment of the silicon grain boundaries, J. Non-Cryst. Solids 354 (2008) 2310-2313. 